\title{
INTERVENIENTES DA TRANSPARÊNCIA ORÇAMENTÁRIA PÚBLICA A NÍVEL NACIONAL ${ }^{1}$
}

\section{INTERVENERS OF TRANSPARENCY PUBLIC BUDGET ON NATIONAL LEVEL}

\section{INTERVENTORES DE LA TRANSPARENCIA PRESUPUESTARIA PÚBLICA A ESCALA NACIONAL}

Anderson de Oliveira Reis, Mestre em Administração pela Universidade Federal de Viçosa (UFV). Endereço Profissional: Universidade Federal de Juiz de Fora/Campus Avançado de

Governador Valadares - Avenida Dr. Raimundo Monteiro Rezende, 330 - Centro

Governador Valadares/MG - CEP 35010-177. Telefone: 333301 1000. URL da Homepage: http://www.ufjf.br/contabeisgv/. Email: anderson.reis@ufjf.edu.br

Marco Aurélio Marques Ferreira, Doutor em Economia Aplicada pela Universidade Federal de Viçosa (UFV). Endereço Profissional: Departamento de Administração e Contabilidade Universidade Federal de Viçosa Av. Peter Henry Rolfs s/n - Campus Universitário CEP: 36570000 - Viçosa/MG. Telefone: (31) 3899-2886. URL da Homepage: http://www.posadministracao.ufv.br/. E-mail: maurelio@ufv.br

Marjorie Angélica Sabioni Ferreira, Mestre em Administração pela Universidade Federal de Viçosa (UFV). Endereço Profissional: R. Presidente Getúlio Vargas, 1856, Centro, Gurupi/TO. CEP: 77402-090. Telefone: (31) 3899-2886. URL da Homepage: http://www.posadministracao.ufv.br/. Email: marjorie_sabioni@yahoo.com.br

\section{RESUMO}

Esse trabalho buscou avaliar os fatores determinantes na variação dos níveis de transparência orçamentária em âmbito nacional, em diversos países. O estudo tem como marco teórico pesquisas nacionais e internacionais com relação a Transparência e Dados Abertos Governamentais. Para atingir os objetivos, foi realizada uma pesquisa descritiva com abordagem quantitativa. Para determinar as variáveis que influenciam nos níveis de transparência dos países foram utilizados os métodos de correlação de Pearson e a Regressão Linear Múltipla. Os resultados demonstram que o nível de educação, a corrupção e a adoção das normas internacionais de contabilidade aplicadas ao setor público (IPSAS) tem um poder de explicação conjunto de $63,8 \%$ das variações no nível de transparência. O que reforça a necessidade de melhorias na qualidade da educação, o esforço de redução da corrupção e o aumento na qualidade das normas de contabilidade pública, visando promover maior transparência orçamentária governamental, com efeitos positivos sobre a qualidade da democracia e o fortalecimento do Estado de direito.

Palavras chave: Transparência; Gestão Pública; Corrupção.

\section{ABSTRACT}

This study evaluated the determinants of variation in budget transparency stages at the national level in several countries. The study's theoretical framework national and international research concerning the Transparency and Government Open Data. To achieve

\footnotetext{
${ }^{1}$ Artigo submetido em 08/09/2016, revisado em 26/12/2016, aceito em 31/03/2017 e divulgado em 01/07/2017 pelo Editor João Carlos Hipólito Bernardes do Nascimento, após double blind review.
}

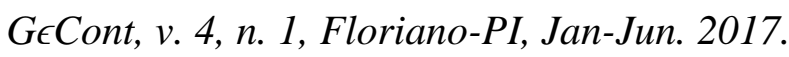


the objectives, a descriptive research was conducted using a quantitative approach. To determine the variables that influence the levels of transparency of countries have used the Pearson correlation methods and Multiple Linear Regression. The results showed that the level of education, corruption and the adoption of international accounting standards applied to the public sector (IPSAS) has an explanatory power range of $63.8 \%$ of the variations in the level of transparency. This reinforces the need for improvements in the quality of education, the effort to reduce corruption and increase the quality of public accounting standards, to promote greater government budget transparency, with positive effects on the quality of democracy and the strengthening of the state of right.

Keywords: Transparency; Public Manangment; Corruption.

\section{RESUMEN}

Este estudio evaluó los factores determinantes de la variación en los niveles de transparencia presupuestaria a nivel nacional en varios países. El estudio tiene como marco teórico la investigación nacional e internacional relativo a la Transparencia y Gobierno Abierto de Datos. Para lograr los objetivos, una investigación descriptiva se llevó a cabo utilizando un enfoque cuantitativo. Para determinar las variables que influyen en los niveles de transparencia de los países han utilizado los métodos de correlación de Pearson y regresión lineal múltiple. Los resultados muestran que el nivel de educación, la corrupción y la adopción de normas internacionales de contabilidad aplicado al sector público (IPSAS) tiene un rango de potencia explicativa del 63,8\% de las variaciones en el nivel de transparencia. Esto refuerza la necesidad de mejoras en la calidad de la educación, el esfuerzo por reducir la corrupción y aumentar la calidad de las normas de contabilidad pública, para promover una mayor transparencia en el presupuesto del gobierno, con efectos positivos sobre la calidad de la democracia y el fortalecimiento del estado de derecho.

Palabras Clave: Contabilidad Pública; Convergencia Internacional; Panorama histórico.

\section{INTRODUÇÃO}

A s discussões sobre transparência ainda podem ser consideradas recentes, 1 tendo se iniciado após a adoção de práticas gerenciais na administração 1 pública da Inglaterra. Esse conceito ganhou maior expressão somente na última década do século $\mathrm{XX}$, quando passou a ser incorporada na agenda governamental (HEALD, 2003). A partir de então, países que buscam promover o processo democrático de acesso às informações sobre a gestão pública têm abordado de forma crescente os debates sobre a transparência.

Assim, a transparência e outros elementos como accountability e participação cidadã começaram a ter elevado destaque na administração pública. Denhardt (2011) considera que esse novo modelo de gestão pública é marcado por uma reinvenção da ação governamental em que há a busca pela descentralização para empoderar os atores locais, estimular a participação da sociedade civil, impulsionado pelo desenvolvimento de tecnologias que facilitam a comunicação e fortalecem a relação Estado-sociedade. Filgueiras (2011) destaca que após a crise internacional de 1970 houve maior engajamento da sociedade no controle da administração pública, e por isso teve um crescimento da demanda por maiores níveis de transparência. Tal demanda é intensificada pelo desenvolvimento das novas Tecnologias de Informação e Comunicação (TICs), especialmente da internet.

O desenvolvimento das TICs permitiu avanços para promoção da transparência e comunicação entre governo e sociedade, tornando o processo mais simples, uma vez que possibilitaram acesso rápido e fácil aos dados governamentais. A ampliação do uso da internet 
e dos sistemas de informações deram início ao movimento que ficou conhecido como Open Data Government ou Dados Governamentais Abertos, em que os governos passaram a publicar informações sobre as ações governamentais em seus websites, principalmente relatórios financeiros.

Diante desse contexto de maior demanda por transparência no setor público e do crescente emprego das TICs para disponibilização de informações governamentais, levanta-se o seguinte questionamento: "Com qual intensidade que aspectos socioeconômicos e demográficos podem explicar os níveis de transparência orçamentária no governo de diferentes países?" O objetivo desse estudo é analisar com qual intensidade aspectos relacionados à atividade econômica, localização geográfica, condição demográfica, acesso à internet, e, percepção da corrupção no setor público podem explicar a transparência orçamentária de diferentes países. Assim, procura-se, com base na literatura sobre o tema, testar a hipótese de que fatores sociais (educação), econômicos (renda), e demográficos (urbanização), dentre outros, possuem ou não, poder de explicação sobre o nível de transparência orçamentária dos países analisados.

Diversos estudos têm abordado as temáticas transparência, dados governamentais abertos e iniciativas de e-government. Como exemplo, pode-se citar Hanberger (2009), que faz reflexões sobre a transparência no governo democrático e, Grimmelikhuijsen e Welch (2012), que abordam os fatores determinantes da transparência de governos locais em websites. Já o estudo de Evans (2012) questiona o processo de governança como forma de promoção da transparência no setor público, e o trabalho de Renzio e Masud (2011) avaliaram os efeitos da transparência nas contas públicas sobre a governança.

No entanto, apesar da quantidade significativa de estudos que abragem a temática, Grimmelikhuijsen e Welch (2012) destacam que a literatura sobre transparência ainda é fragmentada e pouco desenvolvida. Enquanto que Alshawi e Alalwany (2009) consideram que a avaliação de governo eletrônico e das iniciativas para a promoção da transparência é uma área pouco explorada em termos de desenvolvimento e de gestão.

Neste sentido, tornam-se relevantes estudos voltados para a compreensão dos aspectos intervinientes da transparência no intuito de propor mecanismos que contribuam para melhorar o desempenho do setor público, e consequentemente elevar o desenvolvimento dos países por intermédio da inserção da sociedade e maior controle da administração pública.

\section{CONTEXTUALIZAÇÃO TEÓRICA}

Nesta seção, desenvolveu-se a revisão bibliográfica dos conceitos e fundamentos necessários para compreender a dinâmica da transparência e dos dados abertos governamentais, a fim de construir o embasamento teórico para a interpretação dos dados.

\subsection{TRANSPARÊNCIA GOVERNAMENTAL}

É crescente o número de estudos sobre transparência tanto a nível nacional quanto internacional. No Brasil observa-se que a preocupação central é a transparência financeira ou fiscal, seja de órgãos públicos ou de entes da federação como retratado nos estudos de Cruz e Ferreira (2008); Santana Junior et al., (2009); Rausch e Soares, (2010); Cruz et al., (2012); e, Ribeiro e Zuccolotto, (2014).

No âmbito internacional a transparência é considerada por pesquisadores em administração pública como um valor democrático que fortalece a confiança e a boa governança, aumenta o desempenho, torna os governos mais responsáveis e reduz a corrupção (HOOD, 2006; MEIJER, 2009; GRIMMELIKHUIJSEN; WELCH, 2012).

Ribeiro e Zuccolotto (2014) buscaram identificar quais fatores influenciam o nível de 
transparência eletrônica dos municípios no Brasil. Os autores destacaram que a transparência fiscal ainda é incipiente na administração pública brasileira, e, está associada a melhores indicadores de desenvolvimento socioeconômico e fiscal, como saúde, educação, trabalho, renda e receita orçamentária.

A Transparência das Contas Públicas (TCP) buca impedir a prática da corrupção na gestão pública através da influência do controle social, isto é, o controle exercido pela sociedade sobre as ações do Estado. Dessa forma, a TCP pode ser considerada um meio de informação social que deve ser adequado para a compreensão do público-alvo. Neste sentido são traçadas reflexões sobre a necessidade de atuação da sociedade, o que pode fortalecer a accountability dos gestores públicos perante aos cidadãos (RAUSCH; SOARES, 2010).

Cruz et al. (2012), verificaram o nível de transparência das informações acerca da gestão pública municipal e quais características e indicadores socioeconômicos dos municípios influenciam o nível de transparência observado. Os autores destacaram a existência de associação entre as condições socioeconômicas dos municípios e os níveis de transparência na divulgação de informações acerca da gestão pública, observados nos sites dos grandes municípios brasileiros que compuseram a amostra do estudo.

Grimmelikhuijsen e Welch (2012) desenvolveram e testaram empiricamente um modelo teórico que explica os determinantes da transparência em governos locais com relação a informações ambientais divulgadas em websites. Os autores destacam que o nível de transparência está associado com a atenção da mídia, pressão de grupos externos, influência política e capacidade organizacional dos governos locais.

O acesso à informação é fator fundamental para a promoção da transparência. Governo de países que possuem leis de acesso à informação, infraestrutura de telecomunicações, iniciativas de e-government, imprensa livre e maior renda, tendem a ser percebidos como mais transparentes pelos cidadãos (RELLY; SABHAWAL, 2008).

A educação é um dos fatores potenciais que devem ser estudados como determinante para as demandas por maior transparência segundo a pesquisa de Piotrowski e Van Ryzin (2007), que associaram o maior o nível de escolaridade da população com capacidade de exercício do controle social e, por consequência, maior a transparência governamental.

Cassell e Mullaly (2013) encontraram evidências nos municípios dos EUA de que quanto maior o nível de educação e urbanização da população, maior a possibilidade da existência de um sítio eletrônico que disponibilize informações governamentais. Para os autores, quanto melhor a educação da população, maior a probabilidade da utilização de sites sofisticados e de interpretação das informações divulgadas. Neste sentido, a educação é positivamente associada aos níveis de transparência pública.

Os papéis desempenhados pela transparência e confiança na relação entre a corrupção governamental e a satisfação dos cidadãos com os serviços públicos foram os objetos de estudo de Park e Blenkinsopp (2011), que apontaram a relação entre corrupção e satisfação como moderado pela transparência e parcialmente mediada pela confiança.

Menores níveis de corrupção são associados ao desenvolvimento de melhores estruturas de governança que tem como consequência direta maior nível de divulgação de informações públicas (GRIMMELIKHUIJSEN; WELCH, 2012). Dessa maneira, a redução da corrupção pode ser associada a avanços no nível de transparência.

Welch (2012) considera que a participação está positivamente associada à transparência, mas a transparência não leva à participação. As organizações que estão sob forte influência de atores externos apresentam níveis mais elevados de participação, mas em alguns casos, os níveis elevados de influência externa diminui a transparência.

Meijer (2009) afirma que a transparência tem perspectiva moderna sobre a mudança social principalmente com o avanço das TICs, uma vez que, essas tecnologias permitem as pessoas melhor acesso à informação, contribuindo para a racionalização da sociedade. Dessa 
forma, quanto maior a acessibilidade da população a internet, maior será a probabilidade do aumento da transparência no setor público.

Mecanismos de transparência estruturados possibilitam impacto nas políticas públicas com relação a eficiência, a equidade e a responsabilidade democrática. Por isso, barreiras contra a transparência devem ser evitadas, sejam as intrínsecas a realização da atividade pública ou aquelas constituídas por atores externos ao governo (HEALD, 2012).

Os estudos sobre transparência, em âmbito nacional e internacional, buscam entender a temática sobre diferentes perspectivas, no entanto, parece haver um consenso que esse é um elemento fundamental para aperfeiçoar a administração pública, além de promover o desenvolvimento por meio da accountability e controle social nos diferentes níveis de governo.

\subsection{GOVERNO ABERTO E TRANSPARÊNCIA}

A discussão acerca da transparência impulsionou o movimento em direção aos Dados Governamentais Abertos (DGA). Vaz, Ribeiro e Matheus (2011) relatam que com o desenvolvimento das TICs, as bases de dados brutas passaram a ser livremente manipuladas, filtradas ou cruzadas, constituindo-se em novas aplicações e conhecimentos para a sociedade.

DGA ou governo aberto pode ser entendido como as práticas de disponibilização, por meio da internet, das informações e dados governamentais a cerca da gestão pública para a livre utilização pela sociedade (AGUNE; GREGORIO FILHO; BOLLIGER, 2010). A utilização das TICs para a divulgação de informações governamentais trouxe para administração pública novos termos como e-governo e e-democracia, empregados para definir as novas formas de comunicação entre Estado e sociedade.

No intuito de entender o desenvolvimento dessas novas formas de comunicação no setor público, foram desenvolvidos estudos como o de Lee, Chang e Berry (2011), que analisaram os determinantes do desenvolvimento e difusão do e-governo e da e-democracia em 131 países. Como variáveis explicativas foram utilizadas a política de aprendizagem da mudança, as normas políticas, competição e as pressões dos cidadãos. As quatro características são fortemente relacionados em países com alto desenvolvimento da política de e-governo. Já o desenvolvimento da e-democracia de um país está ligado a aspectos internos complexos, tais como normas políticas e pressões do cidadão.

Os portais de governo eletrônico devem ser vistos como canais para fornecer dados e serviços governamentais, e também como ferramenta para a troca de informações e conhecimentos entre diferentes atores sociais e entidades governamentais no intuito de permitir a participação cidadã nos esforços de tomada de decisões coletivas sobre assuntos importantes de interesse público (SANDOVAL-ALMAZAN; GIL-GARCIA, 2012). Dessa forma, os portais se tornaram ferramentas de promoção da transparência e da accountability.

A utilização da internet como meio de comunicação tende a reduzir a diferença de recursos entre os especialistas formuladores de políticas públicas e os cidadãos. No entanto, a tecnologia em si não é suficiente. Fatores exógenos, como características pessoais, ambiente de decisão e aspectos institucionais são determinantes para que a internet tenha impacto positivo na atuação do cidadão (YANG; LAN, 2010).

Nos estudos realizados sobre as TICs, pode-se observar que o e-governo e a utilização da internet são vistos como fatores potenciais para aumentar a transparência e a participação no setor público. Desta forma, há uma tendência de fortalecimento da relação entre Estado e sociedade, buscando o aumento da colaboração entre os atores públicos e os cidadãos para melhorar a gestão governamental. Vários estudos tem sido desenvolvidos visando compreender quais são as melhores alternativas de inclusão da sociedade por intermédios das TICs para promoção do controle social. 
No Brasil, o foco dos estudos atuais tem sido compreender a relação entre dados abertos e participação cidadã, além do fato de se procurar desvendar o porquê de se experimentar tantas iniciativas de dados abertos em diferentes países do mundo (PRADO; LOUREIRO, 2008; PINHO, 2008).

O progresso nas pesquisas é importante para que grupos da sociedade civil possam se organizar em torno de ações práticas na busca por maior envolvimento dos cidadãos na tomada de decisão pública e também pelo requerimento de maior transparência governamental. Isso vem influenciando a forma como os governos se comportam, pois estão sujeitos, em maior ou menor escala, ao controle dos cidadãos, ou seja, ao conjunto de sanções positivas ou negativas impostas pela sociedade para assegurar a conformidade das condutas dos governantes, em alinhamento com as normas culturais e com os valores estabelecidos (ROSS, 2009).

Khagram, Fung e Renzio, (2013) sintetizam quatro aspectos centrais capazes de explicar a evolução do processo de transparência ao longo das últimas décadas em países democráticos, como o Brasil, sendo eles:

i) O processo de transição política em direção a formas mais democráticas de governo e da administração pública;

ii) As crises fiscais e econômicas que obrigam os governos a criar mecanismos mais eficientes de controle dos gastos públicos e disciplina fiscal;

iii) Os crescentes casos de corrupção no Estado, abrindo espaço para os legisladores introduzir reformas que melhorem o acesso dos cidadãos as contas públicas; e,

iv) As influências externas que promovem normas globais que capacitam os reformadores nacionais e atores da sociedade civil.

O Projeto Dados Abertos para Políticas Públicas na América Latina e Caribe (http://www.od4d.org/pt/) foi um importante avanço para a publicação de dados abertos e a divulgação de experiências dessa natureza nos países latino-americanos, cuja implantação, ainda em processo, é fruto de parceria de diferentes organismos mundiais. Esse projeto visa "contribuir para o desenvolvimento de estratégias de dados abertos que levem à prestação de contas, serviços inovadores e à efetividade de políticas públicas, promovendo uma economia do conhecimento mais inclusiva na América Latina e Caribe" (CGI, 2014).

O envolvimento dos cidadãos por meio da internet no Brasil vem seguindo a tendência mundial de acesso a dados abertos, sendo que três eventos contribuíram muito para essa disseminação: i) A criação do Portal da Transparência pela Controladoria Geral da União (CGU) em 2003; ii) A implementação da Lei de Acesso a Informação, em 2011; e, iii) A criação do portal DADOS do Governo Federal, em 2012.

A valorização dos dados abertos está associado ao nível de participação e controle social. Isto é, a conscientização da sociedade de que ela tem o direito do acesso e compreensão das informações públicas, enquanto componente essencial para promoção do controle social.

\section{PROCEDIMENTOS METODOLÓGICOS}

O presente estudo pode ser caracterizado como descritivo quanto aos seus fins, já que pretende-se descrever e analisar os intervenientes da variação nos níveis de transparência em âmbito nacional. A forma de abordagem é de natureza quantitativa, pois se emprega métodos estatísticos para identificar os aspectos intervenientes dos níveis de transparência dentre as variáveis selecionadas com base na literatura científica.

Dados secundários foram coletados em diferentes bases, sendo o Banco Mundial e o Programa das Nações Unidas para o Desenvolvimento (PNUD), as principais, mas foram utilizados também os dados de duas organizações não governamentais, Open Budget 
Iniciative e International Transparency. A amostra utilizada foram os países para os quais foi calculado o Open Budget Index (OBI), utilizado como proxy de transparência orçamentária, no ano base de 2010. Inicialmente contava-se com 93 países, no entanto, devido à indisponibilidade de dados a amostra foi reduzida para 82, sendo eles: Afghanistan, Bosnia and Herzegovina, Cameroon, Equatorial Guinea, Iraq, Papua New Guinea, Saudi Arabia, Sudan, Timor-Leste, Trinidad and Tobago, Vietnam e Yemen.

No intuito de quantificar a influência das variáveis explicativas na variação dos níveis de transparência entre os países estudados, foram realizadas análises de correlação de Pearson e regressão linear múltipla, tendo como ferramenta de apoio o software Statistical Package for the Social Science (SPSS versão 21.0).

A regressão linear múltipla na percepção de Fávero et al., (2009) tem como intuito compreender como a variável dependente $(Y)$ é afetada por outras variáveis $\left(X_{1}, X_{2}, \ldots, X_{n}\right)$ consideradas explicativas. O número de variáveis explicativas a serem inseridas no modelo de regressão depende da experiência e bom senso do pesquisador, que deve apresentar justificativa teórica para inserção de cada variável. Com a finalidade de explicar a variação nos níveis de transparência dos países, foram testadas algumas variáveis, em que a expectativa teórica são apresentadas no Quadro 1.

Quadro 1 - Descrição das variáveis do modelo

\begin{tabular}{|c|c|c|}
\hline Variável & Descrição & Expectativa Teórica \\
\hline OBI & $\begin{array}{l}\text { Índice de Abertura Orçamentária, é o } \\
\text { escore de pontuação de } \\
\text { disponibilização cordo com a } \\
\text { orçamentárias dos países. }\end{array}$ & Variável dependente (Y), sem interpretação. \\
\hline IPC & $\begin{array}{l}\text { Índice de Percepção da Corrupção, é o } \\
\text { escore de pontuação da percepção que a } \\
\text { população tem em relação a corrupção em } \\
\text { seus países. }\end{array}$ & $\begin{array}{l}\text { Positivo, já que a teoria indica que a redução da } \\
\text { corrupção leva a melhoria nas estruturas de } \\
\text { governança, que melhoram os níveis de } \\
\text { transparência. }\end{array}$ \\
\hline TXURB & $\begin{array}{l}\text { Taxa de Urbanização, é a relação entre a } \\
\text { população urbana e a rural. }\end{array}$ & $\begin{array}{l}\text { Positiva, países com maior desenvolvimento } \\
\text { urbano tendem a ser mais transparentes. }\end{array}$ \\
\hline IDH-E & $\begin{array}{l}\text { Índice de Desenvolvimento Humano para } \\
\text { Educação, mede as condições de } \\
\text { desenvolvimento do país em termos de } \\
\text { educação. }\end{array}$ & $\begin{array}{l}\text { Positiva, pois a educação está associada ao nível de } \\
\text { controle social e exigência por transparência. }\end{array}$ \\
\hline DAL & $\begin{array}{l}\text { Dummy que aponta se o país pertence a } \\
\text { America Latina ou não. }\end{array}$ & $\begin{array}{l}\text { Indeterminado, mas há expectativa de que os países } \\
\text { latinos-americanos apresentem comportamento } \\
\text { diferenciado em termos de transparência pública. }\end{array}$ \\
\hline $\ln P I B$ & $\begin{array}{l}\text { Logaritmo natural do Produto Interno } \\
\text { Bruto per capita, usado em forma } \\
\text { logarítmica devido a discrepância entre } \\
\text { seus valores e os das demais variáveis. }\end{array}$ & $\begin{array}{l}\text { Positivo, pois quanto mais desenvolvido } \\
\text { economicamente, maior a probabilidade do país ser } \\
\text { transparente. }\end{array}$ \\
\hline PIAI & $\begin{array}{l}\text { Percentual de Indivíduos com Acesso a } \\
\text { Internet, corresponde ao percentual da } \\
\text { população que possui acesso à internet. }\end{array}$ & $\begin{array}{l}\text { Positiva, pois as TICs facilitam a promoção da } \\
\text { transparência. }\end{array}$ \\
\hline NPP & Número de Partidos Políticos. & $\begin{array}{l}\text { Negativo, de acordo com a teoria, quanto maior for } \\
\text { o número de partidos políticos num país, maior será } \\
\text { a fragilidade da democracia. }\end{array}$ \\
\hline DESPSAUDE & $\begin{array}{l}\text { Percentual do PIB do país que é investido } \\
\text { em saúde. }\end{array}$ & $\begin{array}{l}\text { Positivo, pois quanto melhor as condições de vida } \\
\text { da população, maiores as exigências com o setor } \\
\text { público, e maior a transparência. }\end{array}$ \\
\hline IPSAS & $\begin{array}{l}\text { Dummy que indica se o país adotou ou não } \\
\text { as normas de contabilidade aplicadas ao } \\
\text { setor público até o ano de } 2008 \text {. }\end{array}$ & $\begin{array}{l}\text { Positiva, as normas melhoram a qualidade das } \\
\text { informações a serem evidenciadas no setor público. }\end{array}$ \\
\hline
\end{tabular}




\begin{tabular}{|c|l|l|}
\hline TRAB & $\begin{array}{l}\text { Percentual da população acima de 15 anos } \\
\text { que possui emprego formal. }\end{array}$ & $\begin{array}{l}\text { Positiva, pois quanto maior o nível de emprego há } \\
\text { expectativa de maior desenvolvimento e } \\
\text { consequentemente maior cobrança do governo por } \\
\text { melhor aplicação dos recursos públicos. }\end{array}$ \\
\hline
\end{tabular}

Fonte: Resultados da pesquisa.

O modelo será estimado pelo método dos mínimos quadrados ordinários (MQO) cujo objetivo é obter a menor soma de quadrados dos resíduos possível. Neste método, a equação que melhor se ajusta aos dados é aquela para a qual a diferença entre os valores estimados é menor (CORRAR; PAULO; DIAS FILHO, 2007).

Para a construção do melhor modelo de regressão será utilizado o método stepwise. Para Maroco (2003) os procedimentos de seleção de variáveis apresentam vantagem de indicar, com base num critério exato, quais as variáveis que apresentam relações mais fortes com a variável dependente e por isso são melhores candidatas ao modelo definitivo.

A seguir são apresentados e discutidos os resultados encontrados nessa pesquisa. Primeiramente realiza-se a análise exploratória dos dados com o propósito de conhecer o comportamento das variáveis utilizadas. Já a segunda parte abrange os resultados da regressão linear múltipla, aplicado para analisar as variáveis intervenientes da transparência orçamentária pública nos países.

\section{RESULTADOS E DISCUSSÕES}

\subsection{ANÁLISE EXPLORATÓRIA DOS DADOS (AED)}

A fim de conhecer o comportamento das variáveis estudadas com relação às medidas de centro, distribuição, dispersão e outliers foi realizada a AED. Os valores das estatísticas descritivas são apresentados na Tabela 1 .

A variável OBI assumiu valores entre 0 (Chad) e 92 (South Africa) com média 45,24. Os valores de variância e desvio padrão demonstra significativa discrepância entre os países analisados. A distribuição é levemente assimétrica à esquerda, praticamente mesocúrtica. Já o IPC apresentou valores entre 17 (Chad) e 93 (New Zealand) sendo a média 37,30. A variância e o desvio padrão também evidenciaram a discrepância na percepção de corrupção entre os países. A distribuição é assimétrica à direita e mesocúrtica.

Tabela 1 - Estatística descritiva das variáveis estudadas

\begin{tabular}{lccccccccccc}
\hline & OBI & IPC & PIAI & NPP & $\begin{array}{c}\text { Ln } \\
\text { PIB }\end{array}$ & URB & TRAB & IDHE & DESPSAU & IPSAS & DAL \\
\hline Média & 45,24 & 37,3 & 32,24 & 16,21 & 8,33 & 54,59 & 64,03 & 7,01 & 6,93 & 0,81 & 0,19 \\
Mediana & 49,5 & 32 & 29,84 & 11 & 8,38 & 55,43 & 63,65 & 7,22 & 6,81 & 1 & 0 \\
Moda & 57 & 24 & 10 & 11 & 5,8 & 12,43 & 61,9 & 6,83 & 2,92 & 1 & 0 \\
Desvio-Padrão & 23,43 & 17,66 & 25,06 & 17,4 & 1,36 & 21,97 & 10,24 & 1,53 & 2,51 & 0,39 & 0,39 \\
Variância & 549,12 & 311,76 & 628,01 & 302,93 & 1,85 & 482,62 & 104,8 & 2,36 & 6,3 & 0,15 & 0,15 \\
Assimetria & $-0,21$ & 1,56 & 0,67 & 3,84 & 0,08 & $-0,25$ & 0,21 & $-0,39$ & 1,09 & $-1,63$ & 1,63 \\
Curtose & $-0,57$ & 1,93 & $-0,43$ & 18,13 & $-0,75$ & $-0,89$ & $-0,09$ & $-0,07$ & 2,82 & 0,68 & 0,68 \\
Amplitude & 92 & 76 & 92,67 & 119 & 5,56 & 80,88 & 47 & 7,28 & 14,74 & 1 & 1 \\
Mínimo & 0 & 17 & 0,72 & 1 & 5,8 & 12,43 & 42,3 & 2,72 & 2,92 & 0 & 0 \\
Máximo & 92 & 93 & 93,39 & 120 & 11,36 & 93,31 & 89,3 & 10 & 17,66 & 1 & 1 \\
\hline
\end{tabular}

Fonte: Resultados da pesquisa.

O PIAI tem valores entre 0,72 (Democratic Republic of the Congo) e 93,39 (Norway) com média de 32,24. As medidas de dispersão demonstram a existência de grandes diferenças em relação a acessibilidade da internet entre os países. A distribuição é levemente assimétrica 
à direita e com formato mesocúrtico. O NPP varia entre 1 e 120 com média 16,21. A dispersão mostra distinções significativas no número de partidos políticos entre os países. A distribuição é assimétrica a direita e com formato leptocúrtico.

O $\operatorname{lnPIB}$ teve variação entre 5,8 e 11,36 com média 8,33. Essa variável foi calculada com base no logaritmo natural para reduzir a discrepância entre seus valores e das demais variáveis, por isso não são relevantes a interpretação das suas medidas de dispersão e distribuição.

A TXURB assumiu valores entre 12,43 e 93,31 com média 54,59. A distribuição é levemente assimétrica à esquerda com formato mesocúrtico. Os valores de variância e desvio padrão mostram discrepância no nível de urbanização com países predominantemente urbanos e outros predominantemente rurais, como é o caso de Venezuela, que é praticamente urbano, enquanto que o Uganda é basicamente rural. Já o Brasil apresenta o valor 84.34 podendo ser considerado um país predominantemente urbano.

A variável TRAB apresentou valores entre 42,30 e 89,30, com média 64,30. As medidas de dispersão indicam que não há grande diferença de empregabilidade entre os países, e, as medidas de distribuição indicam que o seu formato se aproxima da normal. $\mathrm{O}$ IDH-E teve valores entre 2,72 e $10 \mathrm{com}$ média 7,01. Não há grandes diferenças no desenvolvimento da educação entre os países analisados. A distribuição nesse caso é levemente assimétrica à esquerda e apresenta formato mesocúrtico.

A DESPSAU variou entre 2,92 e 17,66 com média 6,93. Os valores de dispersão indicam pequenas diferenças entre os países. A distribuição é assimétrica à direita e com formato leptocúrtico. A análise de outliers revelou que dois países eram outliers extremos devendo ser excluídos da análise, sendo eles a África do Sul e a Arábia Saudita. Dessa forma, a amostra final foi composta por 81 países.

\subsection{ASPECTOS INTERVENIENTES DA TRANSPARÊNCIA PÚBLICA ORÇAMENTÁRIA}

Para estimar o modelo de regressão linear múltipla primeiramente foi calculada a correlação entre as variáveis, uma vez que esse é um dos pressupostos básicos do modelo. A Tabela 2 apresenta os valores das correlações entre as variáveis.

Os valores da correlação indicam as primeiras variáveis que devem entrar no modelo de regressão. Nesse caso a variável que apresentou a maior correlação foi IDH-E e, portanto deve ser a primeira a ser inserida no modelo a ser construído. Os valores também indicam as variáveis que não possuem correlação significativa e, portanto, não devem fazer parte do modelo final apesar da expectativa teórica, sendo elas NPP, LnPIB, TRAB e a dummy de localização DAL.

Tabela 2 - Correlação de Pearson

\begin{tabular}{ccc}
\hline & OBI & Sig. \\
\hline OBI & Correlação de Pearson & \\
IPC & 1 & 0,000 \\
PIAI & $0,670^{* *}$ & 0,000 \\
NPP & $0,644^{* *}$ & 0,745 \\
InPIB & 0,037 & 0,416 \\
TXURB & 0,093 & 0,000 \\
TRAB & $0,485^{* *}$ & 0,011 \\
IDHE & $-0,285$ & 0,000
\end{tabular}

G€Cont, v. 4, n. 1, Floriano-PI, Jan-Jun. 2017. 


$\begin{array}{ccc}\text { DESPSAU } & 0,432^{* *} & 0,000 \\ \text { IPSAS } & 0,548^{* *} & 0,000 \\ \text { DAL } & -0,023 & 0,841\end{array}$

Nota: ** Indica que a correlação é significativa no nível 0,01 (2 extremidades). * Indica que a correlação é significativa no nível 0,05 (2 extremidades).

Fonte: Resultados da pesquisa.

O método stepwise foi usado para estimar o modelo de regressão, sendo sugerido três modelos, no qual o terceiro possuiu o maior poder de explicação, conforme resultados apresentados na Tabela 3. O coeficiente de determinação ( $\mathrm{R}^{2}$ ajustado) de 0,638 mostra que $63,8 \%$ das variações no nível de transparência dos países são explicados pela variação conjunta das variáveis escolhidas pelo modelo. Cabe ressaltar que o $\mathrm{R}^{2}$ ajustado é o melhor instrumento de análise do coeficiente de determinação, principalmente em modelos com mais de uma variável independente, uma vez que $\mathrm{R}^{2}$ tende a ser influenciado pela dimensão da amostra e pela dispersão existente nos dados.

Tabela 3 - Modelos de regressão múltipla obtidos pelo método stepwise

\begin{tabular}{ccccc}
\hline Modelo & Variáveis Preditoras & R & R2 & R2 ajustado \\
\hline 1 & IDH-E & 0,727 & 0,528 & 0,522 \\
2 & IDH-E; IPC & 0,772 & 0,596 & 0,585 \\
3 & IDH-E; IPC; IPSAS & 0,808 & 0,652 & 0,638 \\
\hline
\end{tabular}

Fonte: Resultados da pesquisa.

O poder explicativo da proxy de educação está coerente com as expectativas teóricas de Piotrowski e Van Ryzin (2007), Cruz et al., (2012), Cassell e Mullaly (2013) e Wright (2014). Esses autores consideram que a educação tem influência positiva sobre a transparência, uma vez que, quanto maior o nível de educação da população maior é sua capacidade de exercício do controle social, e, de acesso, utilização e interpretação das informações governamentais disponibilizadas de sítios eletrônicos. Dessa forma, há indícios de que os investimentos em educação podem melhorar a gestão pública a longo prazo devido ao aumento da consciência política e do controle social.

A capacidade explicativa da proxy de percepção da corrupção (IPC) atende as expectativas teóricas de Hood (2006), Meijer (2009) e Grimmelikhuijsen e Welch, (2012). Para esses autores a redução na corrupção gera melhorias nos mecanismos de governança pública que tem como consequência direta o aumento nos níveis de transparência e também dos níveis de confiança da sociedade nos governos. Sendo assim, observa-se uma relação estabelecida entre transparência e percepção da corrupção, de forma que quanto menor o nível de percepção da corrupção pela população, maior o nível de transparência pública desse país, reforçando o pensamento de que a transparência é antidoto contra a corrupção.

O poder de explicação da variável IPSAS está de acordo com as expectativas teóricas de Oliveira, Silva e Moraes (2008) e Carvalho et al., (2012). Segundo esses autores a adoção das Normas Internacionais de Contabilidade Aplicadas ao setor público melhora a qualidade tanto das normas quanto das informações a serem divulgadas pelos entes públicos, tornandoas mais compreensíveis aos cidadãos. Destaca-se, que a adoção das normas internacionais de contabilidade, aparentemente está cumprindo um dos seus objetivos que é a instrumentalização do controle social.

Esperava-se que as demais variáveis contribuíssem com o poder de explicação do modelo. As variáveis taxa de urbanização e PIB per capita foram validadas empiricamente por Wright (2014) em estudos sobre transparência em nível municipal. No entanto, essas expectativas não se confirmaram neste estudo a nível nacional.

As demais variáveis se constituíam em expectativas teóricas sem comprovações empíricas que comprovem a relação destas variáveis com os níveis de transparência dos 
países. Com o propósito de validar os resultados encontrados efetuou-se a análise dos pressupostos do modelo. Primeiramente foi realizado o teste F-Anova para analisar a hipótese de $\mathrm{R}^{2}$ ser igual a zero. Os resultados são apresentados na Tabela 4.

Tabela 4 - Teste F: ANOVA

\begin{tabular}{ccccc}
\hline Modelo & & Soma dos Quadrados & F & Sig. \\
\hline \multirow{2}{*}{$\mathbf{1}$} & Regressão & 21673,183 & 85,176 & 0,000 \\
& Resíduos & 19338,305 & & \\
& Total & 41011,487 & & \\
\hline \multirow{2}{*}{$\mathbf{2}$} & Regressão & 24440,493 & 55,309 & 0,000 \\
& Resíduos & 16570,994 & & \\
& Total & 41011,487 & 46,292 & 0,000 \\
\multirow{3}{*}{$\mathbf{3}$} & Regressão & 26755,11 & & \\
& Resíduos & 14256,377 & & \\
\hline
\end{tabular}

Fonte: Resultados da pesquisa.

Verifica-se na Tabela 4 que a significância é menor que $0,01 \%$, por isso rejeita-se a hipótese de que o coeficiente de determinação seja igual a zero. Dessa forma, pelo menos uma das variáveis independentes exerce influência sobre a variação nos níveis de transparência.

Cabe ressaltar a necessidade de se efetuar testes de suposições para as variáveis separadas e em conjunto, e cada modelo apresenta seu conjunto de suposições e pressupostos. Dessa forma, tem-se que os principais pressupostos da regressão linear múltipla são: normalidade dos resíduos, homocedasticidade dos resíduos, ausência de autocorrelação serial dos resíduos e multicolinearidade entre as variáveis independentes (CORRAR; PAULO; DIAS FILHO, 2007; FARIA et al, 2010).

Para testar a normalidade dos resíduos foi utilizado o teste Kolmogorov-Smirnov, que examina se determinada série está conforme a distribuição esperada. O teste KolmogorovSmirnov apresentou o valor de 0,73 com uma significância de 0,2 indicando que não se rejeita a hipótese nula de que se trata de uma distribuição normal, cumprindo-se assim o pressuposto de normalidade.

Para testar a existência de homocedasticidade dos resíduos, foi utilizado o teste Pesarán-Pesarán. Sua forma consiste em se regredir o quadrado dos resíduos padronizados em função do quadrado dos valores estimados padronizados, sendo que se neste estudo foi considerado o nível de significância de 1\%. O resultado do teste é apresentado na Tabela 5.

Tabela 5 - Diagnóstico de homocedasticidade dos resíduos

\begin{tabular}{ccc}
\hline & Soma dos Quadrados & Sig. \\
\hline Regressão & 9,37 & 0,024 \\
Resíduos & 133,726 & \\
\hline
\end{tabular}

Fonte: Resultados da pesquisa.

Após a aplicação do teste, verificou-se que a significância apresentada foi de 2,4\%, o que neste caso indica a rejeição da hipótese nula de existência de homocedasticidade. No intuito de minimizar esse problema o modelo foi estimado com erros padrões robustos. A ausência da autocorrelação serial dos resíduos foi analisada por meio do teste de DurbinWatson que nesse caso assumiu o valor de 2,065. Segundo Corrar et al. (2007), valores próximos de 2 indicam a ausência de correlação serial dos resíduos.

Por último será realizada a análise do diagnóstico de colinearidade, que indica se há correlações entre as variáveis, sob pena de incorrer em problemas na estimação dos 
parâmetros do modelo. Hair et al., (2005), considera que quando as variáveis são multicolineares, fornecem informações semelhantes para explicar e prever determinado fenômeno. O impacto da multicolinearidade é reduzir o poder preditivo de qualquer variável independente na medida em que ela é associada com as demais variáveis. Os valores do diagnóstico de colinearidade são evidenciados na Tabela 6.

Tabela 6 - Diagnóstico de colineariedade

\begin{tabular}{ccc}
\hline Variável & Tolerance & VIF \\
\hline IDH-E & 0,508 & 1,968 \\
IPC & 0,577 & 1,735 \\
IPSAS & 0,794 & 1,259 \\
\hline
\end{tabular}

Fonte: Resultados da pesquisa.

Com base na Tabela 6, percebe-se que o valor tolerance é superior a 0,5 o que, segundo Hair et al. (2005), sugere a ausência de multicolinearidade. Segundo Fávero et al., (2009) os valores de VIF abaixo de 5 também sugerem esta ausência. Dessa forma, comprova-se que o modelo proposto atende aos pressupostos estatísticos de regressão, sendo válido para a previsão das variações dos níveis de transparência entre os países analisados.

$\mathrm{Na}$ Tabela 7 são apresentados os coeficientes beta das variáveis presentes na construção do modelo de regressão linear múltipla. Cabe destacar que, através do teste $\mathrm{T}$ pode-se rejeitar, com nível de significância de $1 \%$, a hipótese de que os coeficientes são iguais a zero.

Tabela 7 - Coeficientes dos fatores determinantes na variação dos níveis de transparência

\begin{tabular}{ccccc}
\hline Variável & Coeficiente $\boldsymbol{\beta}$ & Erro Padrão & t & Sig \\
\hline Constante & $-26,008$ & 7,647 & $-3,401$ & 0,001 \\
IDH-E & 5,862 & 1,447 & 4,051 & 0,000 \\
IPC & 0,433 & 0,117 & 3,691 & 0,000 \\
IPSAS & 15,929 & 4,596 & 3,466 & 0,001 \\
\hline
\end{tabular}

Fonte: Resultados da pesquisa.

Dessa forma, foi possível obter a seguinte equação para previsão da variação dos níveis de transparência entre os países:

$$
O B I=-26,008+5,862 I D H E+0,433 I P C+15,929 I P S A S
$$

Observa-se que em média, o aumento de 1 unidade no indicador de educação aumenta em 5,86\% o nível de transparência. O resultado está de acordo com as expectativas teóricas de que aumentos nos níveis de educação contribuem para o aumento dos níveis de transparência.

A variação de uma unidade no índice de percepção da corrupção aumenta em 0,433\% os níveis de transparência, confirmando a expectativa teórica de que corrupção e transparência estão positivamente associadas. O fato do país ter adotado as Normas Internacionais de Contabilidade Aplicadas ao Setor Público aumenta em 15,92\% os níveis de transparência o que também confirma a teoria de que a qualidade das normas e das informações divulgadas contribuem positivamente para o aperfeiçoamento da transparência.

Em termos gerais, os resultados apresentam consonância com a literatura nacional e internacional, mostrando que a qualidade da educação, a redução da corrupção e o aumento na qualidade regulatória podem aumentar o nível de transparência pública orçamentária, tendo efeitos positivos sobre o desempenho da gestão pública conforme destacado nos relatos de Cruz et al., (2012) e Grimmelikhuijsen e Welch, (2012).

Destaca-se que as variáveis de qualidade da educação e qualidade regulatória são gerenciáveis pelos próprios governos, que podem atuar na qualidade da educação a fim de 
possibilitar que a sociedade tenha capacidade de interpretar as informações divulgadas. É importante ressaltar ainda que, a adoção das normas de contabilidade possibilitam melhor controle do patrimônio público e maior qualidade das informações governamentais divulgadas. Portanto, para aumentar o desempenho da gestão pública deve se considerar esses três aspectos intervenientes da transparência pública orçamentária.

\section{CONSIDERAÇÕES FINAIS}

Os resultados demonstram que, conforme a expectativa teórica, o nível de educação tem uma relação forte com o nível de transparência dos países. A justificativa dessa relação encontra-se no fato de que quando a população possui níveis mais elevados de educação, tende haver maior capacidade de cobrança do governo, de utilização das TICs para acessar aos DGA e ainda de interpretação e utilização dessas informações.

A variável utilizada como proxy de corrupção, também é determinante no nível de transparência dos países. Acredita-se que a redução da corrupção gera melhorias nas estruturas de governança que corroboram para o maior nível de transparência dos países. A variável adoção das normas de contabilidade também se relaciona positivamente com o nível de transparência. Esta relação pode ser explicada pela melhoria na qualidade normativa aplicada aos órgãos públicos responsáveis pela divulgação das informações e também pelo aumento da qualidade dessas informações devido à adoção dessas normas.

Em síntese destaca-se que o modelo foi significativo para explicar a variação no nível de transparência dos países, e indica que melhorias no nível de educação, redução da corrupção e aumento na qualidade normativa da contabilidade pública podem aumentar os níveis de transparência.

Como limitações da pesquisa tem-se que o estudo foi realizado em corte transversal obtendo-se um numero limitado de observações. Além disso, não foi considerado as diferenças entre a democracia dos diferentes países, fator que pode afetar tanto a transparência como a corrupção percebida.

Dessa forma, sugere-se para novos estudos a inclusão de novas variáveis que possam ser utilizadas como proxy de nível ou força da democracia de cada país, além disso, a realização do estudo com dados em painel no intuito de aumentar o numero de observações e o poder explicativo do modelo utilizado.

\section{Referências Bibliográficas}

AGUNE, Roberto Meizi; GREGORIO FILHO, Alvaro Santos; BOLLIGER, Sergio Pinto.

Governo aberto SP: disponibilização de bases de dados e informações em formato aberto. In: Congresso CONSAD de Gestão Pública, Anais..., Brasília, 2010.

ALSHAWI, Sarmad; ALALWANY, Hamid. E-government evaluation: Citizen's perspective in developing countries. Information Technology for Development, v. 15, n. 3, p. 193-208, 2009.

CARVALHO, Frederico A. et al. Fatores Determinantes da Abertura Orçamentária Pública: Um Estudo Empírico Apoiado em Dados Secundários Para uma Amostra de Países. Contabilidade Vista \& Revista, v. 23, n. 4, p. 157-183, 2013.

CASSELL, Mark K.; MULLALY, Sarah. When Smaller Governments Open the Window A Study of Web Site Creation, Adoption, and Presence among Smaller Local Governments in Northeast Ohio. State and Local Government Review, v. 44, n. 2, p. 91-100, 2012.

CGI (Comite Gestor da Internet no Brasil). W3C Brasil cria portal sobre Dados Abertos na

GєCont, v. 4, n. 1, Floriano-PI, Jan-Jun. 2017. 
América Latina. In: http://www.cgi.br/noticia/w3c-brasil-cria-portal-sobre-dados-abertos-naamerica-latina/307. Acesso em 27/04/2014.

CORRAR, Luiz J.; PAULO, Edilson; DIAS FILHO, José Maria. Análise multivariada: para os cursos de administração, ciências contábeis e economia. São Paulo: Atlas, 2007.

CRUZ, Cláudia Ferreira et al. Transparência da gestão pública municipal: um estudo a partir dos portais eletrônicos dos maiores municípios brasileiros. Revista de Administração Pública, v. 46, n. 1, p. 153-76, 2012.

DENHARDT, Robert B. Teorias da administração pública. Cengage Learning, 2011.

EVANS, Mark. Beyond the integrity paradox-towards 'good enough'governance?. Policy Studies, v. 33, n. 1, p. 97-113, 2012.

Faria, Evandro. R. et al., Fatores determinantes na variação dos preços dos produtos contratados por pregão eletrônico. Revista de Administração Pública, v.44, n.6, p14051428, 2010.

FÁVERO, Luiz Paulo et al. Análise de Dados: modelagem multivariada para tomada de decisão. Rio de Janeiro: Campus. 2009.

FILGUEIRAS, Fernando. Além da transparência: accountability e política da publicidade.

Lua Nova, v. 84, p. 65-94, 2011.

GRIMMELIKHUIJSEN, Stephan G.; WELCH, Eric W. Developing and testing a theoretical framework for computer-mediated transparency of local governments. Public

Administration Review, v. 72, n. 4, p. 562-571, 2012.

HAIR, Joseph F. et al. Análise multivariada de dados. Bookman Editora, 2009.

HANBERGER, Anders. Democratic Accountability in Decentralised Governance.

Scandinavian Political Studies, v. 32, n. 1, p. 1-22, 2009.

HEALD, David. Fiscal transparency: concepts, measurement and UK practice. Public Administration, v. 81, n. 4, p. 723-759, 2003.

HEALD, David. Why is transparency about public expenditure so elusive?. International Review of Administrative Sciences, v. 78, n. 1, p. 30-49, 2012.

Hood, Christopher. Transparency in historical perspective. In: Hood, Christopher.; Heald, David. (orgs.) Transparency: the key to better governance? Oxford: Oxford University Press, 2006.

KHAGRAM, Sanjeev; FUNG, Archon; RENZIO, Paolo. Open budgets: The political economy of transparency, participation, and accountability. Brookings Institution Press, 2013.

LEE, Chung-pin; CHANG, Kaiju; BERRY, Frances Stokes. Testing the development and diffusion of e-government and e-democracy: A global perspective. Public Administration Review, v. 71, n. 3, p. 444-454, 2011.

MARÔCO, João. Análise Estatística. Lisboa: Silabo, 2003.

MEIJER, Albert. Understanding modern transparency. International Review of Administrative Sciences, v. 75, n. 2, p. 255-269, 2009.

OLIVEIRA, Robson Ramos; SILVA, Aline Moura Costa da; MORAES, Melissa Christina Corrêa de. Transparência do orçamento governamental dos países: um estudo acerca da associação entre IAO, IDH, PIB e IPSAS. Pensar Contábil, v. 10, n. 42, 2008.

PARK, Heungsik; BLENKINSOPP, John. The roles of transparency and trust in the

GєCont, v. 4, n. 1, Floriano-PI, Jan-Jun. 2017. 
relationship between corruption and citizen satisfaction. International Review of Administrative Sciences, v. 77, n. 2, p. 254-274, 2011.

PINHO, José Antônio Gomes. Investigando portais de governo eletrônico de estados no Brasil: muita tecnologia, pouca democracia. Revista de Administração Pública, v. 42, n. 3 , p. 471-493, 2008.

PIOTROWSKI, Suzanne J.; VAN RYZIN, Gregg G. Citizen attitudes toward transparency in local government. The American Review of Public Administration, v. 37, n. 3, p. 306-323, 2007.

PRADO, Otávio; LOUREIRO, Maria Rita Garcia. Governo eletrônico e transparência: avaliação da publicização das contas públicas das capitais brasileiras. Revista Alcance, v. 13, n. 3, p. 355-372, 2008.

RAUSCH, Rita Buzzi; SOARES, Maurélio. Controle social na administração pública: a importância da transparência das contas públicas para inibir a corrupção. Revista de Educação e Pesquisa em Contabilidade, v. 4, n. 3, p. 23-43, 2010.

RELLY, Jeannine E.; SABHARWAL, Meghna. Perceptions of transparency of government policymaking: A cross-national study. Government Information Quarterly, v. 26, n. 1, p. 148-157, 2009.

RENZIO, Paolo; MASUD, Harika. Measuring and promoting budget transparency: The open budget index as a research and advocacy tool. Governance, v. 24, n. 3, p. 607-616, 2011.

RIBEIRO, Clarice Pereira de Paiva; ZUCCOLOTTO, Robson. A face oculta do Leviatã: transparência fiscal nos municípios brasileiros e suas determinantes socioeconômicas e fiscais. Enfoque: Reflexão Contábil, v. 33, n. 1, p. 37-52, 2014.

ROSS, Edward Alsworth. Social control: A survey of the foundations of order. Transaction Publishers, 2009.

SANDOVAL-ALMAZAN, Rodrigo; GIL-GARCIA, J. Ramon. Are government internet portals evolving towards more interaction, participation, and collaboration? Revisiting the rhetoric of e-government among municipalities. Government Information Quarterly, v. 29, p. S72-S81, 2012.

Santana Junior, Jorge José Barros et al., Transparência Fiscal Eletrônica: uma Análise dos Níveis de Transparência Apresentados nos Sites dos Poderes e Órgãos dos Estados e do Distrito Federal do Brasil. Revista de Educação e Pesquisa em Contabilidade, v.3, n.3, p62-84, 2009.

VAZ, José Carlos; RIBEIRO, Manuella Maia; MATHEUS, Ricardo. Dados governamentais abertos e seus impactos sobre os conceitos e práticas de transparência no Brasil. Cadernos PPG-AU/UFBA, v. 9, n. 1, 2010.

WELCH, Eric W. The relationship between transparent and participative government: A study of local governments in the United States. International Review of Administrative Sciences, v. 78, n. 1, p. 93-115, 2012.

WRIGHT, Gabriel Aragão. Análise dos fatores determinantes da transparência fiscal ativa nos municípios brasileiros. 2014. 123f. Dissertaçãode (Mestrado em Ciências Contábeis), Universidade de Brasília, Brasília, 2013.

YANG, Lihua; LAN, G. Zhiyong. Internet's impact on expert-citizen interactions in public policymaking - A meta analysis. Government information quarterly, v. 27, n. 4, p. 431441, 2010.

GєCont, v. 4, n. 1, Floriano-PI, Jan-Jun. 2017. 